\title{
Changes in stroke volume induced by lung recruitment maneuver can predict fluid responsiveness during intraoperative lung- protective ventilation in prone position
}

Ryota Watanabe, Koichi Suehiro ${ }^{*}$, Akira Mukai, Katsuaki Tanaka, Tokuhiro Yamada, Takashi Mori and Kiyonobu Nishikawa

\begin{abstract}
Background: The present study aimed to evaluate the reliability of hemodynamic changes induced by lung recruitment maneuver (LRM) in predicting stroke volume (SV) increase after fluid loading (FL) in prone position.

Methods: Thirty patients undergoing spine surgery in prone position were enrolled. Lung-protective ventilation (tidal volume, 6-7 mL/kg; positive end-expiratory pressure, $\left.5 \mathrm{cmH}_{2} \mathrm{O}\right)$ was provided to all patients. LRM $\left(30 \mathrm{cmH}_{2} \mathrm{O}\right.$ for 30 s) was performed. Hemodynamic variables including mean arterial pressure (MAP), heart rate, SV, SV variation (SVV), and pulse pressure variation (PPV) were simultaneously recorded before, during, and at $5 \mathrm{~min}$ after LRM and after FL ( $250 \mathrm{~mL}$ in $10 \mathrm{~min}$ ). Receiver operating characteristic curves were generated to evaluate the predictability of SV, PPV, and SV decrease by LRM ( $\triangle S V_{L R M}$ ) for SV responders (SV increase after FL > 10\%). The gray zone approach was applied for $\triangle S V_{\text {LRM }}$.

Results: Areas under the curve (AUCs) for $\triangle S V_{\text {LRM }}, S V$, and PPV to predict SV responders were 0.778 (95\% confidence interval: 0.590-0.909), 0.563 (0.371-0.743), and 0.502 (0.315-0.689), respectively. The optimal threshold for $\triangle S_{\text {LRM }}$ was $30 \%$ (sensitivity, $92.3 \%$; specificity, $70.6 \%$ ). With the gray zone approach, the inconclusive values ranged 25 to $75 \%$ for $\triangle S V_{\text {LRM }}$ (including $50 \%$ of enrolled patients).

Conclusion: In prone position, LRM-induced SV decrease predicted SV increase after FL with higher reliability than traditional dynamic indices. On the other hand, considering the relatively large gray zone in this study, future research is needed to further improve the clinical significance.
\end{abstract}

Trial registration: UMIN Clinical Trial Registry UMIN000027966. Registered 28th June 2017.

Keywords: Lung recruitment maneuver, Prone position, Fluid responsiveness, Stroke volume

\section{Background}

Perioperative fluid therapy is a routine part of clinical practice for most anesthesiologists, but there are several challenges. One of the most complex aspects of

*Correspondence: suehirokoichi@yahoo.co.jp

Department of Anesthesiology, Osaka City University Graduate School

of Medicine, 1-5-7 Asahimachi, Abeno-ku, Osaka 545-8586, Japan perioperative fluid therapy is determining how much fluid should be administered in each patient. Optimal fluid management improves perioperative outcomes, $[1$, 2] while excessive or inadequate infusion can increase the morbidity $[3,4]$. Therefore, it is an issue of great importance to find an appropriate parameter to predict fluid responsiveness [5]. Static indices, including central venous pressure, pulmonary capillary wedge pressure, original author(s) and the source, provide a link to the Creative Commons licence, and indicate if changes were made. The images or other third party material in this article are included in the article's Creative Commons licence, unless indicated otherwise in a credit line to the material. If material is not included in the article's Creative Commons licence and your intended use is not permitted by statutory regulation or exceeds the permitted use, you will need to obtain permission directly from the copyright holder. To view a copy of this licence, visit http://creativecommons.org/licenses/by/4.0/. The Creative Commons Public Domain Dedication waiver (http://creativeco mmons.org/publicdomain/zero/1.0/) applies to the data made available in this article, unless otherwise stated in a credit line to the data. 
and global end-diastolic volume index, have traditionally been used for volume assessment but are of limited value in predicting fluid responsiveness [6]. In contrast, dynamic indices, such as stroke volume variation (SVV) and pulse pressure variation (PPV), have recently been used and are superior to static indices in discriminating fluid responders $[7,8]$. However, the reliability of these indices is below the clinically acceptable level in patients with low tidal volume (less than $8 \mathrm{~mL} / \mathrm{kg}$ of body weight) and in those with low airway driving pressure (less than $\left.20 \mathrm{~cm} \mathrm{H}_{2} \mathrm{O}\right)[9,10]$.

Lung-protective ventilation, defined as lower tidal volume $(6-7 \mathrm{~mL} / \mathrm{kg})$ and positive end-expiratory pressure (PEEP) $\left(5-10 \mathrm{~cm} \mathrm{H}_{2} \mathrm{O}\right)$, is becoming a standard of care for surgical patients and can improve the outcomes of postoperative patients $[11,12]$. In this situation, the usefulness of dynamic indices in predicting fluid responsiveness is limited, reducing their applicability in daily clinical practice [13]. Lung recruitment maneuver (LRM) is a fundamental technique in lung-protective ventilation to reopen the lung alveoli. Furthermore, LRM induces hemodynamic changes including a transient decrease in venous return and stroke volume (SV). A previous study showed that LRM-induced SV changes can predict SV increase after volume expansion, even in patients undergoing lung-protective ventilation [14]. However, the usefulness of LRM in assessing volume status in prone position has not been evaluated. Prone positioning, which is often employed in neurosurgical surgery, represents a challenging setting under this circumstance owing to the possibility of massive bleeding [15]. In addition, prone positioning is associated with SV reduction induced by vena caval compression and increased intrathoracic pressure [16]. Therefore, identification of fluid responders with respect to SV in this setting is a major concern for anesthesiologists. Lung-protective ventilation is commonly used in prone position; thus, under this condition, the usefulness of dynamic indices is limited, as described in a previous report [16].

The present study aimed (1) to evaluate the reliability of LRM-induced hemodynamic changes in predicting SV increase after fluid loading in prone position and (2) to compare the predictability of this indicator for fluid responsiveness with that of traditional dynamic indices in patients undergoing lung-protective ventilation in prone position.

\section{Methods}

\section{Anesthetic management}

Ethical approval was given by the Ethical Committee of Osaka City University Graduate School of Medicine, (No. 3693, Chairperson Prof. Tetsuo Arakawa) on 23 February 2017. The current study was registered on the UMIN
Clinical Trials Registry database (registration number: UMIN000027966) before the initial enrolment. Written informed consent was obtained from all patients. In this study, patients undergoing spine surgery in prone position were enrolled. The exclusion criteria were as follows: those younger than 20 years and those who had atrial fibrillation, symptomatic cerebrovascular disease, and/or reduced cardiac function (ejection fraction $<50 \%$ ).

No premedication was provided. Each patient consumed clear liquids until $3 \mathrm{~h}$ before surgery. Anesthesia was induced with propofol, remifentanil, and rocuronium. After securing the airway, lung-protective mechanical ventilation was employed with a tidal volume of $6-7 \mathrm{~mL} / \mathrm{kg}$ of ideal body weight and PEEP of $5 \mathrm{~cm} \mathrm{H}_{2} \mathrm{O}$. The ventilation rate was adjusted to maintain end-tidal carbon dioxide between 35 and $40 \mathrm{mmHg}$. General anesthesia was maintained using sevoflurane, desflurane, or propofol and remifentanil. An arterial line was inserted into the radial artery, which was connected to a Vigileo ${ }^{\mathrm{TM}}$ / FloTrac ${ }^{\mathrm{TM}}$ system monitor (Edwards Lifesciences, Irvine, CA, USA). Anesthetic depth was controlled to maintain the bispectral index between 45 and 60 (BIS Vista ${ }^{\mathrm{TM}}$ monitoring system; Aspect Medical Systems, Natick, MA, USA). After anesthetic induction, patients were turned to the prone position laying on four pads to relieve pressure to the abdomen.

\section{Measurement of SV and SVV}

SV and SVV were calculated using the Vigileo-FloTrac system, which continuously measures hemodynamic variables by analyzing arterial pressure waveforms from a standard peripheral arterial catheter without the need for external calibration. The mechanism has been described previously in detail [17].

\section{PPV calculation}

Pulse pressure (PP) was defined as the difference between diastolic and systolic arterial pressures. As previously described, [18] PPV was calculated as follows: $\mathrm{PPV}=($ maximum $\mathrm{PP}-$ minimum $\mathrm{PP}) /[$ (maximum $\mathrm{PP}+$ minimum $\mathrm{PP}$ ) / 2].

\section{Study protocol}

The design of the study is shown in Fig. 1. The study protocol was performed under stable hemodynamic conditions, which was defined as follows: mean arterial pressure (MAP) and heart rate (HR) changes within $\pm 10 \%$ in 1 min before measurements $[19,20]$. Just before changing position from supine to prone (T0), hemodynamic variables including MAP, HR, SV, SVV, and PPV were recorded (in supine position). Ten minutes after prone positioning, LRM (continuous airway pressure of $30 \mathrm{cmH}_{2} \mathrm{O}$ for $30 \mathrm{~s}$ ) was performed, and hemodynamic 


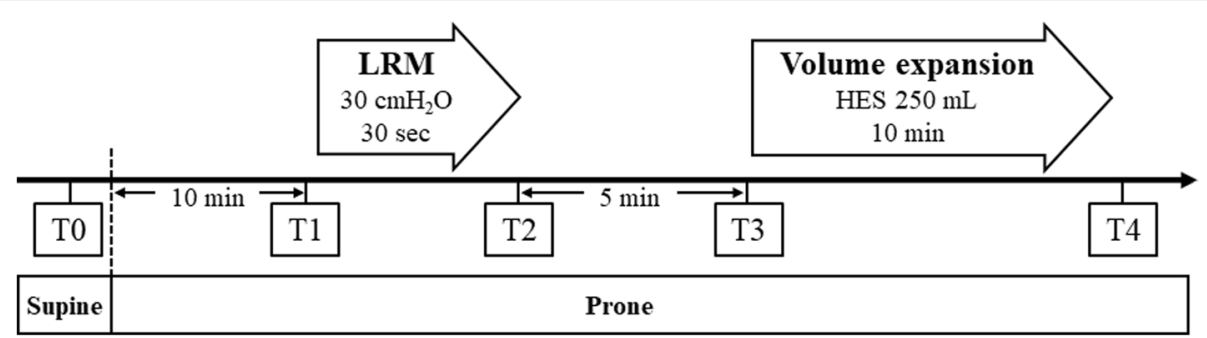

Fig. 1 Study protocol. LRM consisted of applying a continuous positive airway pressure of $30 \mathrm{~cm} \mathrm{H}_{2} \mathrm{O}$ for $30 \mathrm{~s}$. Volume expansion consisted of an infusion of $250 \mathrm{~mL}$ HES given in $10 \mathrm{~min}$. Five sets of hemodynamic measurements including mean arterial pressure, heart rate, stroke volume, stroke volume variation, and pulse pressure variation were performed. T0: baseline measurement in supine position; T1: baseline measurement in prone position 10 min after turning prone and before LRM; T2: at the end of LRM; T3: second baseline measurement 5 min after LRM; T4: after volume expansion. Abbreviations: LRM, lung recruitment maneuver; HES, hydroxyethyl starch

variables simultaneously recorded before LRM (T1), at the end of LRM (T2), and at 5 min after LRM (T3). The LRM setting in the present study was decided in accordance with a previous study [14]. After recording, volume expansion was performed using $250 \mathrm{~mL}$ of Voluven ${ }^{\circledR}$ (hydroxyethyl starch; Otsuka Pharmaceutical Co. Ltd., Tokyo, Japan) in $10 \mathrm{~min}$. After fluid administration (T4), hemodynamic variables were also recorded. SVV and PPV variables at the time points of T0 and T3 were defined as $\mathrm{SVV}_{\text {supine }} / \mathrm{PPV}_{\text {supine }}$ (T0), and $\mathrm{SVV}_{\text {prone }} /$ $\mathrm{PPV}_{\text {prone }}$ (T3), respectively.

\section{Statistical analysis}

The primary endpoint was set as the reliability of LRMinduced SV change and SVV for fluid responsiveness, with comparison performed using receiver operating characteristic (ROC) analysis. In a previous report, the AUC of $\triangle S V_{L R M}$ to discriminate fluid responders in supine position was 0.96 [14]. In a previous study investigating the ability of SVV to discriminate fluid responders in patients undergoing lung-protective ventilation in prone position, the AUC of SVV was reported as 0.53 [16]. Referring to these studies, we hypothesized that AUCs for $\triangle S_{\text {LRM }}$ and SVV would be 0.9 and 0.6 , respectively. We conducted the power analysis as the following; $\mathrm{HO}: \mathrm{AUC}=0.6$ versus $\mathrm{H} 1$ : $\mathrm{AUC}$ of $\Delta \mathrm{SV}_{\mathrm{LRM}}>0.6$ with $\alpha=0.05$ and power $=0.80$, assuming that the ratio of responders and non-responders would be 1:2. The ratio of responders and non-responders was determined referring to the previous report [21]. According to the power analysis, a sample size was calculated as 27 patients. Considering a drop-out rate of $10 \%$, finally, we planned to include 30 patients in this study.

Correlations between percentage change in SV by LRM $\left(\Delta \mathrm{SV}_{\mathrm{LRM}}\right)$ and percentage change in SV after fluid loading $\left(\Delta \mathrm{SV}_{\mathrm{FL}}\right)$ was examined using Pearson correlation coefficient. Furthermore, $\Delta S V_{\mathrm{LRM}}$ and $\Delta \mathrm{SV}_{\mathrm{FL}}$ were compared using the four-quadrant analysis. In the four-quadrant plot analysis, $\Delta \mathrm{SV}_{\mathrm{LRM}}$ and $\Delta \mathrm{SV}_{\mathrm{FL}}$ were drawn in the four quadrants. The results of this analysis were assessed using the concordance rate, which was defined as the percentage of data points located in the upper right or lower left corner of the four-quadrant plot. The concordance rate is defined as good when it was more than $92 \%$, as shown by Critchley et al. [22] Patients were divided into two groups (responders and nonresponders with respect to SV) according to percentage increases in SV after fluid loading, with responders defined as those with $\Delta \mathrm{SV}_{\mathrm{FL}}>10 \%$. The definition of responders was in accordance with previous studies [14, 23]. ROC curves were generated for $\mathrm{SVV}, \mathrm{PPV}$, and $\triangle \mathrm{SV}_{\mathrm{LRM}}$ to distinguish responders from nonresponders. In ROC analyses, optimal thresholds and AUCs were calculated. AUCs by ROC analysis were compared using a previously described method [24]. The $p$-values for the ROC analysis were calculated by testing against the hypothesis of $\mathrm{AUC}=0.50$.

Furthermore, we applied the gray zone approach to test the predictive values of $\Delta S V_{L R M}$. The gray zone approach was used to assess the values for which the target variables did not provide definitive information, which has been described previously [14, 25]. Briefly, a two-step procedure was employed in the gray zone approach. First, bootstrap resampling was performed for $\Delta S V_{L R M}$ and $\triangle \mathrm{MAP}_{\mathrm{LRM}}$. The best cut-off thresholds and their 95\% confidence intervals (CI) were calculated from 1000 bootstrapped populations. The optimal threshold was selected to maximize Youden's index (i.e., J= sensitivity + specificity -1$)$. Second, the inconclusive range of each value for the assessment of SV or BP responsiveness (i.e., cut-off values with sensitivity $<90 \%$ or specificity $<90 \%)$ was calculated. If the $95 \% \mathrm{CI}$ of the optimal threshold from the first step was larger than the inconclusive range from the second step, the values from the first step were obtained as gray zone values.

Hemodynamic variables were compared using Student's paired t-test, Mann-Whitney $U$ test, and 
Table 1 Patient characteristics

\begin{tabular}{ll}
\hline Variables & $\begin{array}{l}\text { All patients } \\
(\boldsymbol{n}=\mathbf{3 0})\end{array}$ \\
\hline Gender, $\mathrm{M} / \mathrm{F}$ & $17 / 13$ \\
Age, $\mathrm{yr}$ & $59.9 \pm 17.9$ \\
Height, $\mathrm{cm}$ & $159 \pm 9.8$ \\
Weight, $\mathrm{kg}$ & $59.0 \pm 13.6$ \\
Body Mass index, $\mathrm{kg} / \mathrm{m}^{2}$ & $23.1 \pm 4.6$ \\
ASA physical status, I/I/III/IV & $2 / 23 / 4 / 1$ \\
Comorbidities & \\
Hypertension, $\mathrm{n}$ & 13 \\
Coronary artery disease, $\mathrm{n}$ & 2 \\
Chronic obstructive pulmonary disease, $\mathrm{n}$ & 1 \\
Asthma, $\mathrm{n}$ & 1 \\
Chronic kidney disease, $\mathrm{n}$ & 4 \\
Diabetes, $\mathrm{n}$ & 5 \\
Dyslipidemia, $\mathrm{n}$ & 4 \\
Ventilatory conditions & \\
Tidal volume, ml/kg ideal body weight & $6.29 \pm 0.47$ \\
Respiratory rate, cycles/min & $12.1 \pm 1.0$ \\
Positive endo-expiratory pressure, $\mathrm{cm} \mathrm{H}_{2} \mathrm{O}$ & 5.0 \\
Peak inspiratory pressure, $\mathrm{cm} \mathrm{H}_{2} \mathrm{O}$ & $16.7 \pm 3.9$ \\
End-inspiratory plateau pressure, $\mathrm{cm} \mathrm{H}_{2} \mathrm{O}$ & $11.3 \pm 3.4$ \\
\hline Data are &
\end{tabular}

Data are expressed as mean (SD) or number unless stated

Abbreviations: ASA American Society of Anaesthesiologist

chi-squared test. For all analyses, a $p$-value $<0.05$ was considered statistically significant. Statistical analysis was performed using StatFlex software version 6.0 (Artech Co. Ltd., Osaka, Japan), and SigmaPlot software version 13.0 (Systat Software Inc., San Jose, CA, USA).

\section{Results}

\section{Patient characteristics and hemodynamic data}

Patient characteristics are summarized in Table 1. A total of 30 patients were enrolled in this study. Hemodynamic variables during the study period are shown in Table 2 . There were no complications including prolonged hypotension and fatal arrhythmia during the study. When the patients were turned to the prone position, both of SVV and PPV significantly increased, while SV significantly decreased ( $\mathrm{T} 0$ vs $\mathrm{T} 1$ ). There were no significant changes in HR and MAP between the time points of T0 and T1. LRM induced a significant decrease in MAP and SV in both groups (T1 vs T2). For responders, SV significantly increased after fluid loading (T3 vs T4).

\section{SV changes during the study period}

There were 13 fluid responders with respect to SV. Figure 2 shows the SV change in responders and nonresponders during the study period. The SV decrease during LRM was greater in responders $(33 \pm 12 \mathrm{~mL})$ than in nonresponders $(19 \pm 11 \mathrm{~mL})(p=0.004)$. As shown in Fig. 3, $\Delta \mathrm{SV}_{\mathrm{LRM}}$ were significantly correlated with $\Delta \mathrm{SV}_{\mathrm{FL}}$ $\left(\Delta \mathrm{SV}_{\mathrm{LRM}}: \mathrm{r}=0.609, p<0.001\right)$. The trending ability of $\Delta \mathrm{SV}_{\mathrm{LRM}}$ was examined using four-quadrant analysis

Table 2 Hemodynamic variables in responders $(n=13)$ and nonresponders $(n=17)$ during the study

\begin{tabular}{|c|c|c|c|c|c|c|c|c|}
\hline Variables & T0 & $\mathrm{T} 1$ & $\mathrm{~T} 2$ & T3 & T4 & $\begin{array}{l}P \text { Value } \\
\text { T0 versus T1 }\end{array}$ & $\begin{array}{l}P \text { Value } \\
\text { T1 versus } \mathrm{T} 2\end{array}$ & $\begin{array}{l}P \text { Value } \\
\text { T3 versus T4 }\end{array}$ \\
\hline \multicolumn{9}{|l|}{$\mathrm{HR}(\mathrm{bpm})$} \\
\hline Responders & $69 \pm 12$ & $68 \pm 13$ & $67 \pm 13$ & $69 \pm 15$ & $68 \pm 14$ & 0.428 & 0.732 & 0.231 \\
\hline Nonresponders & $67 \pm 10$ & $66 \pm 10$ & $63 \pm 10$ & $66 \pm 10$ & $66 \pm 9$ & 0.496 & $0.010^{*}$ & 0.744 \\
\hline \multicolumn{9}{|l|}{$\operatorname{MAP}(\mathrm{mmHg})$} \\
\hline Responders & $77 \pm 13$ & $77 \pm 12$ & $58 \pm 13$ & $74 \pm 12$ & $80 \pm 12$ & 0.801 & $<0.001^{*}$ & $0.031^{*}$ \\
\hline Nonresponders & $78 \pm 9$ & $78 \pm 9$ & $66 \pm 13$ & $78 \pm 8$ & $78 \pm 8$ & 0.231 & $<0.001^{*}$ & 0.859 \\
\hline \multicolumn{9}{|l|}{ Stroke volume (mL) } \\
\hline Responders & $62 \pm 14$ & $59 \pm 14$ & $26 \pm 21$ & $56 \pm 14$ & $67 \pm 15$ & $0.002^{*}$ & $<0.001^{*}$ & $<0.001^{*}$ \\
\hline Nonresponders & $59 \pm 11$ & $58 \pm 11$ & $39 \pm 17$ & $58 \pm 11$ & $60 \pm 11$ & $0.009^{*}$ & $<0.001^{*}$ & $0.002^{*}$ \\
\hline \multicolumn{9}{|l|}{ SVV (\%) } \\
\hline Responders & $10 \pm 3$ & $12 \pm 4$ & & $12 \pm 4$ & $10 \pm 2$ & $0.005^{*}$ & & $0.047^{*}$ \\
\hline Nonresponders & $12 \pm 4$ & $13 \pm 6$ & & $13 \pm 5$ & $12 \pm 5$ & $0.046^{*}$ & & 0.067 \\
\hline \multicolumn{9}{|l|}{ PPV (\%) } \\
\hline Responders & $9 \pm 3$ & $10 \pm 4$ & & $10 \pm 5$ & $9 \pm 3$ & $0.015^{*}$ & & 0.057 \\
\hline Nonresponders & $10 \pm 6$ & $11 \pm 6$ & & $11 \pm 5$ & $9 \pm 5$ & $0.037^{*}$ & & $0.008^{*}$ \\
\hline
\end{tabular}

Statistical analysis Paired t-test. Data are expressed as mean \pm SD. * statistically significant $p<0.05$

Abbreviations: BP Blood pressure, HR Heart rate, MAP Mean arterial blood pressure, SVV Stroke volume variation, PPV Pulse Pressure variation, T0, just before changing position from supine to prone; $\mathrm{T} 1$, before recruitment maneuver in prone position; $\mathrm{T} 2$, at the end of recruitment maneuver; $\mathrm{T} 3,5$ min after recruitment maneuver / baseline before volume expansion; T4, after volume expansion 


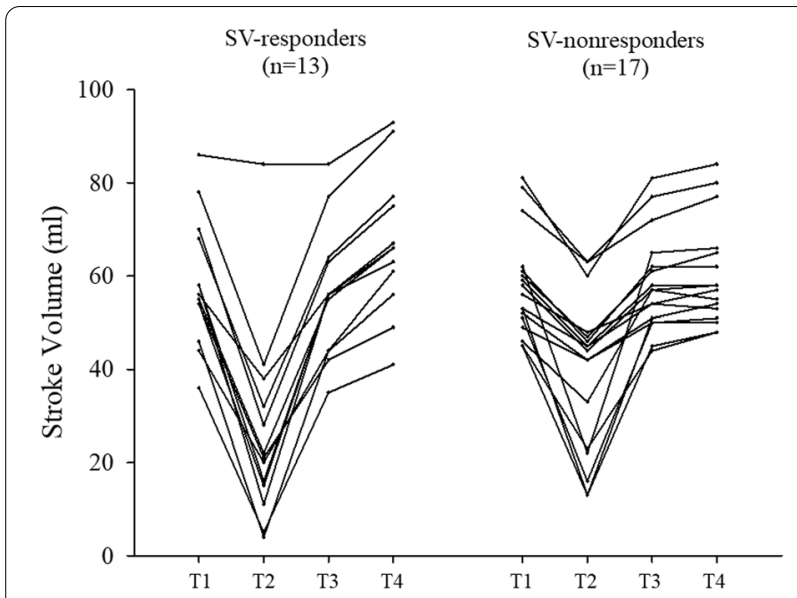

Fig. 2 Change in among responders and nonresponders to fluid loading at points $\mathrm{T} 1$ (before lung recruitment maneuver), $\mathrm{T} 2$ (at the end of lung recruitment maneuver), T3 (at 5 min after lung recruitment maneuver), and T4 (after volume expansion). SV, stroke volume

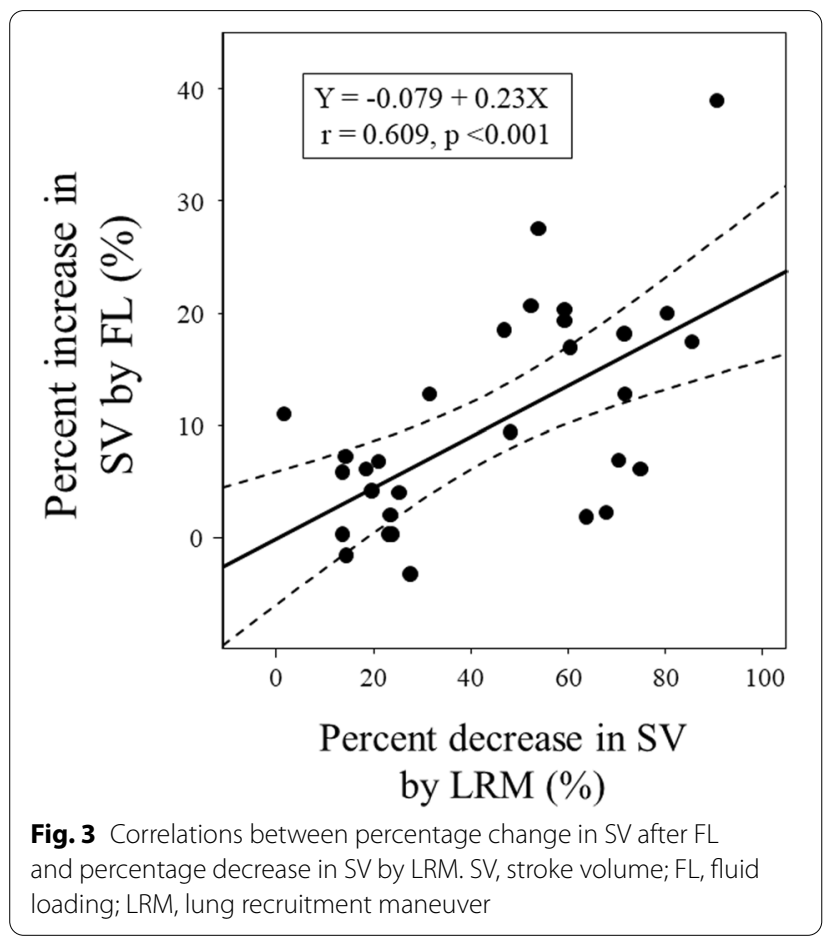

(Fig. 4). In the four-quadrant analysis, the concordance rate was $93.3 \%$.

\section{Predictability of fluid responsiveness with respect to SV}

Figure 5 illustrates the ROC analysis for the assessment of the ability to discriminate fluid responders with respect to SV. The AUCs for $\triangle \mathrm{SV}_{\mathrm{LRM}}, \mathrm{SVV}_{\text {prone, }}$ and $\mathrm{PPV}_{\text {prone }}$ to predict responders were 0.778 (95\% CI: 0.590-0.909),

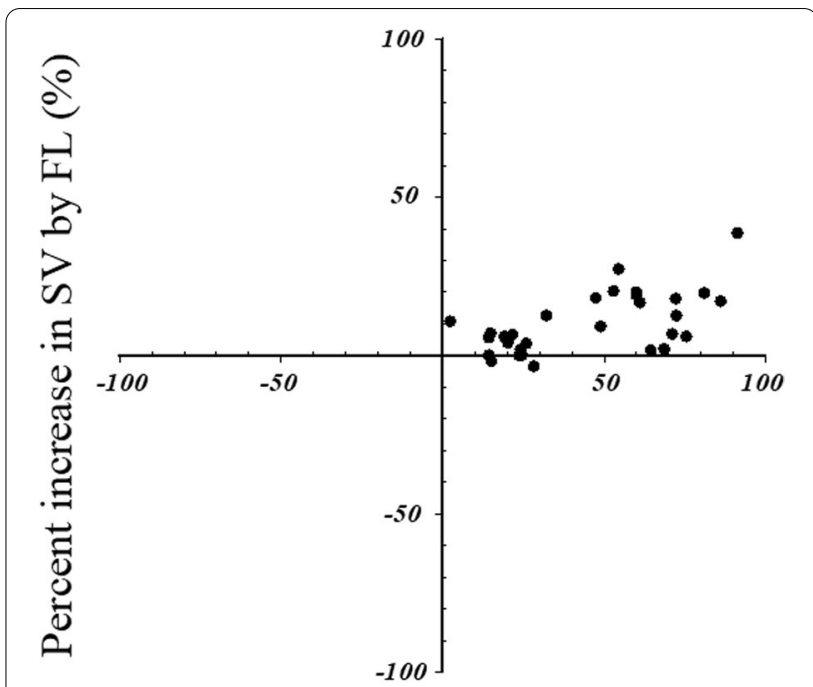

Percent decrease in SV by LRM (\%)

Fig. 4 Four quadrant plot analysis to examine the concordance between percentage change in $\mathrm{SV}$ after $\mathrm{FL}$ and percentage decrease in SV by LRM. SV, stroke volume; FL, fluid loading; LRM, lung recruitment maneuver

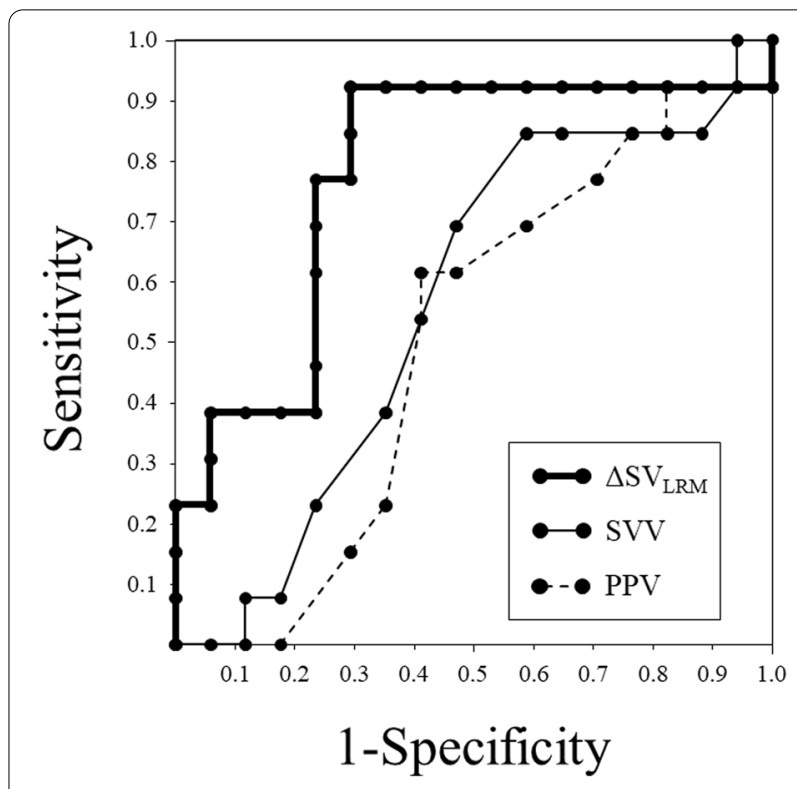

Fig. 5 Receiver operating characteristic curves for $S V_{\text {prone, }} P P V_{\text {prone, }}$ and $\triangle S V_{\text {LRM }}$ to distinguish responders (SV increase after fluid loading $>10 \%)$. SV, stroke volume; BP, blood pressure; $\mathrm{SVV}_{\text {prone, }}$ stroke volume variation at time point T3; $P$ PV $V_{\text {prone, }}$ pulse pressure variation at time point T3; $\triangle S V_{L R M}$, decrease in stroke volume by lung recruitment maneuver

0.563 (95\% CI: 0.371-0.743), and 0.502 (95\% CI: $0.315-$ $0.689)$, respectively. $\Delta \mathrm{SV}_{\mathrm{LRM}}$ showed the best predictability in responders $(p=0.003)$, whereas both $\mathrm{SVV}_{\text {prone }}$ and 
$\mathrm{PPV}_{\text {prone }}$ were not significant predictors in responders ( $p=0.563$ and 0.984 , respectively). The optimal threshold for $\Delta S V_{\text {LRM }}$ was $30 \%$, with a sensitivity of $92.3 \%$ and a specificity of $70.6 \%$. Furthermore, $\mathrm{SVV}_{\text {supine }}$ and $\mathrm{PPV}_{\text {supine }}$ were not significant predictors for SV-responsiveness $\left(\mathrm{SVV}_{\text {supine }}, \mathrm{AUC:}\right.$ 0.611, $p=0.293$; $\mathrm{PPV}_{\text {supine }}, \mathrm{AUC:}$ 0.550, $p=0.646$ ) (Table 3).

With the gray zone approach, the inconclusive range of $\triangle \mathrm{SV}_{\mathrm{LRM}}$ for $\mathrm{SV}$ responders was $25-75 \%$ (including $50 \%$ of enrolled patients; Fig. 6).

\section{Discussion}

In the present study, we investigated the reliability of LRM-induced hemodynamic changes in predicting SV response after fluid administration in patients undergoing lung-protective ventilation in prone position. The SV decrease (30\%) during LRM could predict SV increase after fluid administration. $\triangle \mathrm{SV}_{\mathrm{LRM}}$ was significantly correlated with $\Delta S V_{\mathrm{FL}}$. The predictability of $\Delta S V_{\mathrm{LRM}}$ for fluid responsiveness was better than that of SVV and PPV, which are traditional indicators of fluid responsiveness.

Prone positioning is commonly employed during various surgical procedures, especially brain and spine surgeries, and induces a decrease in chest compliance and an increase in abdominal pressure, which in turn affects hemodynamics such as decreased venous return and stroke volume. Volume expansion is the first-line treatment for $\mathrm{CO}$ reduction during surgery, [26] but inadequate fluid loading in prone position can lead to an increase in edema of the larynx and pharynx, making extubation difficult in the operating room [27, 28]. On the other hand, intraoperative goal-directed fluid therapy based on SV optimization improves postoperative outcomes in surgical patients [29]. Hence, predicting fluid responsiveness in prone position is an issue of major concern among anesthesiologists.
Previous studies $[16,18,30,31]$ investigating the reliability of dynamic indices in assessing fluid responsiveness in prone position have presented various results. Even in the supine position, the reliability of dynamic indices is limited in patients with low tidal volume or low ventilatory driving pressure. In fact, in the study by Min et al. without lung-protective ventilation, SVV served as a good predictor of fluid responsiveness in prone position, with an AUC of 0.78 in the ROC analysis [31]. Conversely, the predictability of SVV became poorer among patients undergoing lung-protective ventilation in prone position, with an AUC of 0.53 [16]. Yonis et al. also showed that $100 \%$ of the patients with lung-protective ventilation in prone position were in the gray zone for PPV [32]. Lungprotective ventilation is becoming a standard for intraoperative management and for improving postoperative outcomes during in prone position [33]. Considering the increasing application of lung-protective ventilation in the surgical population, [34] the applicability of dynamic indices in prone position would diminish. Therefore, a new approach for the assessment of fluid responders in prone position is required.

Intraoperative LRM is often used as part of lung protective ventilation to reduce intraoperative lung collapse, improve oxygenation, and reduce the incidence of postoperative pulmonary complications (PPC) [35]. Xiong et al. showed that intraoperative lung protective ventilation including LRM prevents PPC also in prone spine surgery [33]. LRM increases intrathoracic and transpulmonary pressures, which results in decreased venous return and increased pulmonary vascular resistance, thus leading to decreased SV [14]. LRM-induced hemodynamic collapse depends on volume status. In an experimental study, [36] volume depletion by LRM was significantly higher under hypovolemic condition than under normo- and hypervolemic conditions. Taking this

Table 3 ROC analyses for $\triangle S V_{L R M}, S V_{\text {prone, }} \mathrm{PPV}_{\text {prone, }} \mathrm{SWV}_{\text {supine }}$ and $\mathrm{PPV}_{\text {spine }}$ to discriminate responders

\begin{tabular}{|c|c|c|c|c|c|}
\hline Variables & $\begin{array}{l}\text { Cut-off value } \\
\text { (\%) }\end{array}$ & $\begin{array}{l}\text { AUC } \\
(95 \% \mathrm{Cl})\end{array}$ & $\begin{array}{l}\text { Sensitivity } \\
(\%)\end{array}$ & $\begin{array}{l}\text { Specificity } \\
(\%)\end{array}$ & $P$ value \\
\hline$\Delta S V_{\text {LRM }}(\%)$ & 30 & $\begin{array}{l}0.778 \\
(0.590-0.909)\end{array}$ & 92.3 & 70.6 & $0.003^{*}$ \\
\hline$S V_{\text {prone }}(\%)$ & 13 & $\begin{array}{l}0.563 \\
(0.371-0.743)\end{array}$ & 84.6 & 41.2 & 0.563 \\
\hline$P P V_{\text {prone }}(\%)$ & 9 & $\begin{array}{l}0.502 \\
(0.315-0.689)\end{array}$ & 61.5 & 58.8 & 0.984 \\
\hline $\mathrm{SWV}_{\text {supine }}(\%)$ & 8 & $\begin{array}{l}0.611 \\
(0.417-0.782)\end{array}$ & 38.5 & 82.4 & 0.293 \\
\hline$P P V_{\text {supine }}(\%)$ & 10 & $\begin{array}{l}0.550 \\
(0.358-0.731)\end{array}$ & 84.6 & 35.3 & 0.646 \\
\hline
\end{tabular}

*statistically significant $p<0.05$

Abbreviations: $R O C$ Receiver operating characteristics, SV Stroke volume, $\triangle S V_{L R M}$ Decrease in stroke volume by lung recruitment maneuver, $S V V_{\text {prone }}$ Stroke volume variation at time point T3, $P P V_{\text {prone }}$ Pulse pressure variation at time point T3, SVV supine Stroke volume variation at time point T0, $P P V_{\text {spine }} P$ Pulse pressure variation at time point T0, AUC Area under the curve 


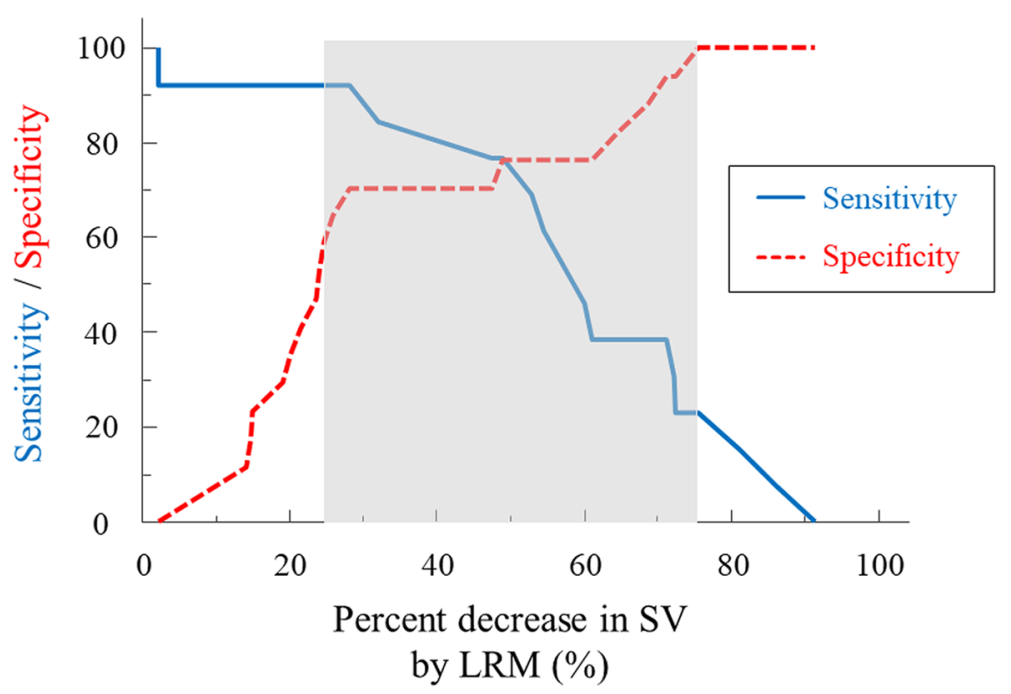

Fig. 6 Gray zone for $\triangle S V_{L R M}$. Blue and red lines denote sensitivity and specificity, respectively. The gray zone indicates the inconclusive range for each variable. $\triangle S V_{\text {LRM }}$, decrease in stroke volume by lung recruitment maneuver; $S V$, stroke volume; LRM, lung recruitment maneuver

into account, Biais et al. [14] applied LRM for the assessment of fluid responsiveness. In their clinical study, a 30\% decrease in SV during LRM could predict SV increase after volume expansion, with a sensitivity of $88 \%$ and a specificity of $92 \%$. In the present study, we investigated the ability of LRM-induced hemodynamic changes to predict fluid responsiveness in prone position. Consistent with the results of the study by Biais et al., [14] even in prone position, a $30 \%$ decrease in $\triangle S V_{\text {LRM }}$ could predict a $10 \%$ increase in $\Delta \mathrm{SV}_{\mathrm{FL}}$ with high sensitivity and specificity ( 92.3 and $70.6 \%$, respectively). On the other hand, the traditional measures of SVV and PPV were not significant discriminators, which is consistent with previous studies. As shown in our study, the predictability of $\triangle \mathrm{SV}_{\mathrm{LRM}}$ for fluid responsiveness was better than that of SVV and PPV among patients in prone position.

Although there were no complications induced by LRM in this study, there is a risk of adverse events such as severe hypotension, fatal arrhythmia, and pneumothorax with LRM procedure $[37,38]$. In the current study, the decrease in MAP induced by LRM (responders: 77 to $58 \mathrm{mmHg}$, nonresponders: 78 to $66 \mathrm{mmHg}$ ) were similar with those in the previous study [14] (LRM was performed in supine position; responders: 71 to $51 \mathrm{mmHg}$, nonresponders: 71 to $55 \mathrm{mmHg}$ ). As indicated by Young et al., [39] LRM should be performed when the patients' oxygen saturation is constantly low (less than 94\%) and following a disconnection from the respiratory circuit. LRM should be considered according to an individual risk-benefit assessment and should not be routinely applied for assessing fluid responsiveness. Anesthesiologists can perform LRM procedures to assess fluid responsiveness when dynamic indices such as SVV and PPV are within the gray zone. In addition, considering the adverse effects induced by LRM, future studies are needed to decide whether $\Delta S V_{\text {LRM }}$ in shorter duration and/or at lower pressure can maintain a high accuracy in discriminating fluid responders.

In this study, the percentage of fluid responders was $43 \%$, which is lower than that (approximately $50 \%$ ) indicated in several systematic reviews about fluid responsiveness $[40,41]$. This difference may be due to the differences in the amount of fluid infused and the definition of fluid responders.

We applied the gray zone approach to determine the inconclusive range for $\Delta \mathrm{SV}_{\mathrm{LRM}}$. The inconclusive range for $\Delta S V_{L R M}$ was $25-75 \%$ (including $50 \%$ of all patients). The inconclusive range was relatively larger for $\Delta \mathrm{SV}_{\mathrm{LRM}}$ than that reported in a previous study investigating it in spine position (22-37\%, including 36\% of enrolled patients). This discrepancy may be due to the large distribution of $\triangle \mathrm{SV}_{\mathrm{LRM}}(2-91 \%)$ in our study. Prone positioning reduces chest compliance, the change of which depends on patients' body constitution. Various changes in chest compliance may lead to a wide range of hemodynamic effects during LRM. Although the predictability of $\triangle \mathrm{SV}_{\mathrm{LRM}}$ for fluid responsiveness was excellent in the ROC analyses, considering the gray zone, these indices should be carefully used in clinical practice.

Our study has some limitations. First, we used the Vigileo/FloTrac system to measure SV. The accuracy of this system in measuring SV depends on systemic vascular resistance, [17] which can be a major limitation. Second, the sample size of this study might be inadequate. In this 
study, we conducted the power analysis using a difference of 0.90 and 0.60 , referring to previous studies. However, if the AUC of 0.75 , which is the threshold for considering a diagnostic test to be accurate [42], was used as the null hypothesis, the sample size would be larger. It is also possible that an increase in the number of enrolled patients would narrow the inconclusive range of $\Delta S V_{L R M}$. Third, the duration of hemodynamic stability of 1 min might be short. However, we decided the definition referred to the previous studies assessing fluid responsiveness [19, 20]. Additionally, as shown in Table 2, HR and MAP at T0, T1 and T3 were quite similar. Therefore, the impact of this limitation on the results is minimized. Fourth, intraoperative use of HES may lead to the incidence of adverse outcomes in abdominal surgery [43]. However, in this study, we administered $250 \mathrm{~mL}$ of HES solely to confirm fluid responsiveness, and do not recommend the continuous administration of HES during surgery. The administration of HES $(250 \mathrm{~mL})$ are frequently used in previous studies assessing fluid responsiveness, [19, 44] and we consider that there may be few adverse effects induced by this infusion. Despite these limitations, the present study showed a new approach for assessing hemodynamic response after volume expansion in prone position.

\section{Conclusions}

In conclusion, in patients undergoing lung-protective ventilation in prone position, LRM-induced SV decrease predicted SV increase after fluid loading with a higher reliability than traditional dynamic indices, including SVV and PPV. On the other hand, considering that $\triangle S V_{\text {LRM }}$ had a relatively large gray zone and there is no consensus on which setting of LRM is particularly effective in predicting fluid responsiveness, it should be further investigated whether $\Delta S V_{L R M}$ in different settings can provide a higher accuracy in discriminating fluid responders. As intraoperative hypervolemia can lead to an increase in postoperative complications, volume loading should be carefully performed according to the need of patients [45]. Routine volume loading should not be given solely based on the presence of fluid responsiveness [45]. Further studies are required to construct a new protocol for goal-directed fluid therapy that takes LRMinduced hemodynamic changes into account.

\footnotetext{
Abbreviations

BP: Blood pressure; CO: Cardiac output; SW: Stroke volume variation; PPV: Pulse pressure variation; PEEP: Positive end-expiratory pressure; LRM: Lung recruitment maneuver; SV: Stroke volume; HR: Heart rate; MAP: Mean arterial pressure; FL: Fluid loading; ROC: Receiver operating characteristic; AUC : Areas under the curve; $\mathrm{Cl}$ : Confidence intervals; ASA: American Society of Anaesthesiologist.
}

Acknowledgements Not applicable.

\section{Authors' contributions}

Study design: KS. Advisor for study protocol and management of the study: KT, TY, TM and KN. Study conduction: RW, KS and AM. Data analysis: RW, KS and KT. Manuscript preparation: RW and KS. Editing and approval of the manuscript: AM, KT, TY, TM and KN. All authors have read and approved the manuscript.

\section{Funding}

Only departmental funds were used for this study.

\section{Availability of data and materials}

The datasets analysed during the current study are available from the corresponding author on reasonable request.

\section{Declarations}

Ethics approval and consent to participate

This study was approved by the Ethics Committee of the Osaka City University Graduate School of Medicine on 23 February 2017 (reference number: 3693), and was performed in accordance with the Declaration of Helsinki. All participating subjects was informed about this study protocol in details and provided written informed consent before enrollment in this study.

\section{Consent for publication}

Not applicable.

\section{Competing interests}

Dr. Suehiro has received speaker fees from Edwards Lifesciences and Otsuka Pharmaceutical Co. Ltd. Other authors have no conflicts of interest to declare.

Received: 26 December 2020 Accepted: 18 November 2021

Published online: 02 December 2021

\section{References}

1. Thacker JK, Mountford WK, Ernst FR, Krukas MR, Mythen MM. Perioperative fluid utilization variability and association with outcomes: considerations for enhanced recovery efforts in sample US surgical populations. Ann Surg. 2016;263(3):502-10.

2. Pearse RM, Harrison DA, MacDonald N, Gillies MA, Blunt M, Ackland G, et al. Effect of a perioperative, cardiac output-guided hemodynamic therapy algorithm on outcomes following major gastrointestinal surgery: a randomized clinical trial and systematic review. JAMA. 2014;311(21):2181-90.

3. Bellamy MC. Wet, dry or something else? Br J Anaesth. 2006;97(6):755-7.

4. Oh TK, Song IA, Do SH, Jheon S, Lim C. Association of perioperative weight-based fluid balance with 30-day mortality and acute kidney injury among patients in the surgical intensive care unit. J Anesth. 2019;33(3):354-63.

5. Navarro LH, Bloomstone JA, Auler JO Jr, Cannesson M, Rocca GD, Gan TJ et al. Perioperative fluid therapy: a statement from the international fluid optimization group. Perioper Med (Lond). 2015:4:3.

6. Guerin L, Monnet X, Teboul JL. Monitoring volume and fluid responsiveness: from static to dynamic indicators. Best Pract Res Clin Anaesthesiol. 2013;27(2):177-85.

7. Suehiro K, Rinka H, Ishikawa J, Fuke A, Arimoto H, Miyaichi T. Stroke volume variation as a predictor of fluid responsiveness in patients undergoing airway pressure release ventilation. Anaesth Intensive Care. 2012:40(5):767-72

8. Cannesson M, Musard H, Desebbe O, Boucau C, Simon R, Henaine R, et al. The ability of stroke volume variations obtained with Vigileo/FloTrac system to monitor fluid responsiveness in mechanically ventilated patients. Anesth Analg. 2009;108(2):513-7. 
9. Suehiro K, Okutani R. Influence of tidal volume for stroke volume variation to predict fluid responsiveness in patients undergoing one-lung ventilation. J Anesth. 2011;25(5):777-80.

10. Muller L, Louart G, Bousquet PJ, Candela D, Zoric L, de La Coussaye JE, et al. The influence of the airway driving pressure on pulsed pressure variation as a predictor of fluid responsiveness. Intensive Care Med. 2010;36(3):496-503.

11. Severgnini P, Selmo G, Lanza C, Chiesa A, Frigerio A, Bacuzzi A, et al. Protective mechanical ventilation during general anesthesia for open abdominal surgery improves postoperative pulmonary function. Anesthesiology. 2013;118(6):1307-21.

12. Futier $E$, Constantin JM, Paugam-Burtz C, Pascal J, Eurin M, Neuschwander $A$, et al. A trial of intraoperative low-tidal-volume ventilation in abdominal surgery. N Engl J Med. 2013;369(5):428-37.

13. Mair S, Tschirdewahn J, Gotz S, Frank J, Phillip V, Henschel B, et al. Applicability of stroke volume variation in patients of a general intensive care unit: a longitudinal observational study. J Clin Monit Comput. 2017;31(6):1177-87.

14. Biais $M$, Lanchon $R$, Sesay $M$, Le Gall L, Pereira B, Futier E, et al. Changes in stroke volume induced by lung recruitment maneuver predict fluid responsiveness in mechanically ventilated patients in the operating room. Anesthesiology. 2017;126(2):260-7.

15. Bacchin MR, Ceria CM, Giannone S, Ghisi D, Stagni G, Greggi T, et al. Goal-directed fluid therapy based on stroke volume variation in patients undergoing major spine surgery in the prone position: a cohort study. Spine. 2016;41(18):E1131-7.

16. Berger K, Francony G, Bouzat P, Halle C, Genty C, Oddoux M, et al. Prone position affects stroke volume variation performance in predicting fluid responsiveness in neurosurgical patients. Minerva Anestesiol. 2015;81(6):628-35.

17. Suehiro K, Tanaka K, Mikawa M, Uchihara Y, Matsuyama T, Matsuura T, et al. Improved performance of the fourth-generation FloTrac/Vigileo system for tracking cardiac output changes. J Cardiothorac Vasc Anesth. 2015;29(3):656-62.

18. Biais M, Bernard O, Ha JC, Degryse C, Sztark F. Abilities of pulse pressure variations and stroke volume variations to predict fluid responsiveness in prone position during scoliosis surgery. Br J Anaesth. 2010;104(4):407-13.

19. Kimura A, Suehiro K, Juri T, Fujimoto Y, Yoshida H, Tanaka K, et al. Hemodynamic changes via the lung recruitment maneuver can predict fluid responsiveness in stroke volume and arterial pressure during one-lung ventilation. Anesth Analg. 2021:133(1):44-52.

20. Mukai A, Suehiro K, Kimura A, Tanaka K, Yamada T, Mori T, et al. Effect of systemic vascular resistance on the reliability of noninvasive hemodynamic monitoring in cardiac surgery. J Cardiothorac Vasc Anesth. 2021;35(6):1782-91.

21. MacDonald N, Ahmad T, Mohr O, Kirk-Bayley J, Moppett I, Hinds CJ, et al. Dynamic preload markers to predict fluid responsiveness during and after major gastrointestinal surgery: an observational substudy of the OPTIMISE trial. Br J Anaesth. 2015;114(4):598-604.

22. Critchley LA, Lee A, Ho AM. A critical review of the ability of continuous cardiac output monitors to measure trends in cardiac output. Anesth Analg. 2010;111(5):1180-92.

23. Cecconi M, Monge Garcia MI, Gracia Romero M, Mellinghoff J, Caliandro F, Grounds RM, et al. The use of pulse pressure variation and stroke volume variation in spontaneously breathing patients to assess dynamic arterial elastance and to predict arterial pressure response to fluid administration. Anesth Analg. 2015;120(1):76-84.

24. DeLong ER, DeLong DM, Clarke-Pearson DL. Comparing the areas under two or more correlated receiver operating characteristic curves: a nonparametric approach. Biometrics. 1988;44(3):837-45.

25. Cannesson M, Le Manach Y, Hofer CK, Goarin JP, Lehot JJ, Vallet B, et al. Assessing the diagnostic accuracy of pulse pressure variations for the prediction of fluid responsiveness: a "gray zone" approach. Anesthesiology. 2011;115(2):231-41.

26. Fellahi JL, Futier E, Vaisse C, Collange O, Huet O, Loriau J, et al. Perioperative hemodynamic optimization: from guidelines to implementation-an experts' opinion paper. Ann Intensive Care. 2021;11(1):58.

27. Li F, Gorji R, Tallarico R, Dodds C, Modes K, Mangat S, et al. Risk factors for delayed extubation in thoracic and lumbar spine surgery: a retrospective analysis of 135 patients. J Anesth. 2014;28(2):161-6.

28. Ramchandran S, Day LM, Line B, Buckland AJ, Passias P, Protopsaltis T, et al. The impact of different intraoperative fluid administration strategies on postoperative Extubation following multilevel thoracic and lumbar spine surgery: a propensity score matched analysis. Neurosurgery. 2019;85(1):31-40.

29. Michard F, Giglio MT, Brienza N. Perioperative goal-directed therapy with uncalibrated pulse contour methods: impact on fluid management and postoperative outcome. Br J Anaesth. 2017;119(1):22-30.

30. Yang SY, Shim JK, Song Y, Seo SJ, Kwak YL. Validation of pulse pressure variation and corrected flow time as predictors of fluid responsiveness in patients in the prone position. Br J Anaesth. 2013;110(5):713-20.

31. Min JJ, Lee JH, Hong KY, Choi SJ. Utility of stroke volume variation measured using non-invasive bioreactance as a predictor of fluid responsiveness in the prone position. J Clin Monit Comput. 2017;31(2):397-405.

32. Yonis H, Bitker L, Aublanc M, Perinel Ragey S, Riad Z, Lissonde F, et al. Change in cardiac output during Trendelenburg maneuver is a reliable predictor of fluid responsiveness in patients with acute respiratory distress syndrome in the prone position under protective ventilation. Crit Care. 2017;21(1):295.

33. Xiong W, Chen P, Gao J, Yuan RX. Lung protective ventilation in elderly patients undergoing spinal operation in the prone position: a randomized controlled trial. Nan Fang Yi Ke Da Xue Xue Bao. 2016;36(2):215-9.

34. Wanderer JP, Ehrenfeld JM, Epstein RH, Kor DJ, Bartz RR, FernandezBustamante A, et al. Temporal trends and current practice patterns for intraoperative ventilation at U.S. academic medical centers: a retrospective study. BMC Anesthesiol. 2015;15:40.

35. Cui Y, Cao R, Li G, Gong T, Ou Y, Huang J. The effect of lung recruitment maneuvers on post-operative pulmonary complications for patients undergoing general anesthesia: a meta-analysis. PLOS One 2019;14(5):e0217405.

36. Nielsen J, Nilsson M, Freden F, Hultman J, Alstrom U, Kjaergaard J, et al. Central hemodynamics during lung recruitment maneuvers at hypovolemia, normovolemia and hypervolemia. A study by echocardiography and continuous pulmonary artery flow measurements in lung-injured pigs. Intensive Care Med. 2006;32(4):585-94.

37. Writing Group for the Alveolar Recruitment for Acute Respiratory Distress Syndrome Trial I, Cavalcanti AB, Suzumura EA, Laranjeira LN, Paisani DM, Damiani LP, et al. Effect of lung recruitment and titrated positive end-expiratory pressure (PEEP) vs low PEEP on mortality in patients with acute respiratory distress syndrome: a randomized clinical trial. JAMA. 2017;318(14):1335-45.

38. Algaba A, Nin N, por el GTIRAdIS. Alveolar recruitment maneuvers in respiratory distress syndrome. Med Int. 2013;37(5):355-62.

39. Young CC, Harris EM, Vacchiano C, Bodnar S, Bukowy B, Elliott RRD, et al. Lung-protective ventilation for the surgical patient: international expert panel-based consensus recommendations. Br J Anaesth. 2019;123(6):898-913.

40. Messina A, Dell'Anna A, Baggiani M, Torrini F, Maresca GM, Bennett V, et al. Functional hemodynamic tests: a systematic review and a metanalysis on the reliability of the end-expiratory occlusion test and of the mini-fluid challenge in predicting fluid responsiveness. Crit Care. 2019;23(1):264.

41. Alvarado Sanchez JI, Caicedo Ruiz JD, Diaztagle Fernandez JJ, Amaya Zuniga WF, Ospina-Tascon GA, Cruz Martinez LE. Predictors of fluid responsiveness in critically ill patients mechanically ventilated at low tidal volumes: systematic review and meta-analysis. Ann Intensive Care. 2021;11(1):28.

42. Ray P, Le Manach Y, Riou B, Houle TT. Statistical evaluation of a biomarker. Anesthesiology. 2010;112(4):1023-40

43. Futier E, Garot M, Godet T, Biais M, Verzilli D, Ouattara A, et al. Effect of Hydroxyethyl starch vs saline for volume replacement therapy on death or postoperative complications among high-risk patients undergoing major abdominal surgery: the FLASH randomized clinical trial. JAMA. 2020;323(3):225-36.

44. Kimura A, Suehiro K, Juri T, Tanaka K, Mori T. Changes in corrected carotid flow time induced by recruitment maneuver predict fluid responsiveness in patients undergoing general anesthesia. J Clin Monit Comput. 2021.

45. Suehiro K. Update on the assessment of fluid responsiveness. J Anesth. 2020;34(2):163-6.

\section{Publisher's Note}

Springer Nature remains neutral with regard to jurisdictional claims in published maps and institutional affiliations. 